\title{
Г.Н. Якушевич
}

\section{Математическая модель активного четырехполюсника корректирующего широкополосного усилителя с обратной связью}

\begin{abstract}
Получена математическая модель активного четырехполюсника корректирующего широкополосного усилителя с неравномерной АЧХ (с подъемом АЧХ с ростом частоты) на основе нового аналитического выражения для коэффициента усиления с учетом нелинейной частотной зависимости АЧХ. Подъем АЧХ происходит плавно с повышением частоты и стремится к линейной зависимости на верхних частотах. Для реализации неравномерной АЧХ используется последовательная и параллельная обратная связь (ОС). Приведены результаты компьютерного моделирования частотных характеристик корректирующего широкополосного усилителя с неравномерной АЧХ. Корректирующий широкополосный усилитель может быть использован для компенсации неравномерности АЧХ (уменьшение АЧХ с ростом частоты) радиоприемных и радиопередающих трактов радиотехнических систем.

Ключевые слова: математическая модель, активный четырехполюсник, корректирующий, широкополосный усилитель, ОС.
\end{abstract}

doi: $10.21293 / 1818-0442-2018-21-4-1-26-32$

Быстродействие современных радиоприемных устройств во многом определяется характеристиками входных каскадов, выполненных на основе широкополосных усилителей. Неравномерность АЧХ (уменьшение АЧХ с ростом частоты) одного каскада широкополосного усилителя может достигать 1-2 дБ в рабочей полосе частот $[1,2]$. При использовании многокаскадных усилителей и с учетом дополнительных потерь на высоких частотах неравномерность АЧХ радиоприемных трактов в радиотехнических системах может достигать 4-6 дБ. При проектировании неравномерность АЧХ широкополосных усилителей компенсируется введением диссипативных корректирующих цепей $[3,4]$. Проектирование проводится на основе генетического алгоритма. Однако проектирование широкополосных усилителей на основе генетического алгоритма не может на начальном этапе определить элементы структурной схемы и их физическое назначение для получения требуемой АЧХ усилителя [5-8].

В данной работе для компенсации неравномерности АЧХ (уменьшение АЧХ с ростом частоты) радиоприемных и радиопередающих трактов используется корректирующий (с подъемом АЧХ с ростом частоты) широкополосный усилитель с ОС.

Параметры рассеяния активного четырехполюсника корректирующего широкополосного усилителя с ОС

На рис. 1 приведена схема усилителя, содержащая активный четырехполюсник и двухполюсник параллельной ОС.

В работах [9, 10] были приведены аналитические выражения параметров рассеяния и номинального коэффициента передачи по мощности для активного четырехполюсника широкополосного усилителя с параллельной ОС с равномерной АЧХ и линейной ФЧХ $\left|S_{210 C}(f)\right|=$ const и $\Theta_{210 C}=\arg \Theta_{210 C}(f)=$ $=180^{\circ}\left(1-f / f_{\mathrm{B}}\right)$, где $f$ - текущая частота, $f_{\mathrm{B}}$ - частота нормировки.
В работе [11] автором была рассмотрена линейная частотная зависимость подъема АЧХ корректирующего усилителя во всем диапазоне частот $\left|S_{21 \mathrm{OC}}(f)\right|=S_{21 \mathrm{OC} 0}\left(1+N\left(f / f_{B}\right)\right)$. Реально же коэффициент усиления увеличивается плавно с повышением частоты и только на верхних частотах принимает линейную зависимость.

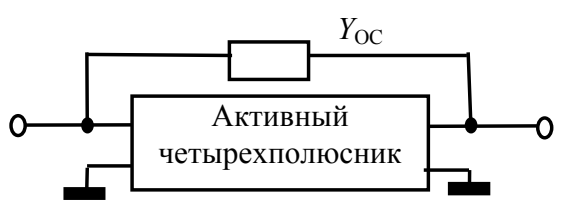

Рис. 1. Схема усилителя с параллельной ОС

В данной работе коэффициент передачи с учетом неравномерности АЧХ определяется выражением

$$
S_{21 \mathrm{OC}}(N(f))=\left|S_{21 \mathrm{OC} 0}\right| \cdot N(f) \cdot \exp \left(j \Theta_{21 \mathrm{OC}}\right),
$$

где $N(f)=\sqrt{1+M(f)^{2}}$ - коэффициент частотных искажений; $M(f)=M_{B} \cdot f / f_{\mathrm{BM}}-$ коэффициент неравномерности; $M_{B}$ - коэффициент неравномерности на верхней частоте $f_{\mathrm{BM}_{\mathrm{B}}} \mathrm{AЧХ,}$ $S_{210 \mathrm{C} 0}=S_{210 \mathrm{C}}\left(f_{\mathrm{BM}}\right) / M_{\mathrm{B}}-$ начальный коэффициент передачи.

Тогда аналитические выражения для коэффициентов отражения по входу $S_{11 \text { Ач}}(N(f))$ и выходу $S_{22 \text { Ач }}(N(f)), \quad$ коэффициента прямой передачи $S_{21 \text { Ач }}(N(f))$, номинального коэффициента передачи по мощности $G_{\text {НомАч}}(N(f))$ активного четырехполюсника корректирующего широкополосного усилителя с параллельной ОС с нелинейной неравномерной АЧХ запишутся в следующем виде:

$$
S_{11 \mathrm{AY}}(N(f))=S_{22 \mathrm{AЧ}}(N(f))=\frac{Y_{\mathrm{OC}}\left(1-S_{21 \mathrm{OC}}(N(f))\right)}{2-Y_{\mathrm{OC}}\left(1-S_{21 \mathrm{OC}}(N(f))\right)},
$$


Г.Н. Якушевич. Математическая модель активного четырехполюсника корректирующего широкополосного усилителя 27

$$
\begin{aligned}
& S_{21 \mathrm{AY}}(N(f))=\frac{2\left(2 S_{21 \mathrm{OC}}(N(f))-Y_{\mathrm{OC}}\left(1-S_{21 \mathrm{OC}}^{2}(N(f))\right)\right.}{\left(2-Y_{\mathrm{OC}}\left(1-S_{21 \mathrm{OC}}(N(f))\right)^{2}\right.}, \\
& G_{\text {HOMAЧ }}(N(f))=\left|S_{21 \mathrm{AY}}(N(f))\right|^{2} /\left(1-\left|S_{11 \mathrm{AY}}(N(f))\right|^{2}\right)^{2},
\end{aligned}
$$

где $Y_{\mathrm{OC}}-$ проводимость двухполюсника параллельной ОС.

Коэффициент неравномерности из выражения для частотных искажений запишется $M(f)=\sqrt{N(f)^{2}-1}$.

В табл. 1 приведены рассчитанные значения коэффициентов неравномерности АЧХ на верхней частоте для коэффициентов частотных искажений 2, 4 и 6 дБ.

Т а бли ц а 1

Значения коэффициентов неравномерности АЧХ

\begin{tabular}{|c|c|c|c|}
\hline$N_{B}$, раз (дБ) & $1,259(2)$ & $1,585(4)$ & $1,995(6)$ \\
\hline$M_{B}$, ра3 & 0,76 & 1,23 & 1,73 \\
\hline
\end{tabular}

В табл. 2-4 для коэффициента передачи $S_{21 \mathrm{OC}}\left(f_{\mathrm{BM}}\right)=8$ дБ и фазы $\Theta_{21 \mathrm{OC}}=-45^{\circ}$ на верхней частоте для заданных значений $\Theta_{210 C}^{\circ}$ от $180^{\circ}$ до $-90^{\circ}$ с шагом $-45^{\circ}$ приведены результаты расчетов $M_{\text {дБ }}, N_{\text {дБ }}, \mid S_{21}$ ОС $\left(N_{\text {дБ }}(f)\right) \mid$ для коэффициентов частотных искажений на верхней частоте 2, 4, 6 дБ.

Табли ца 2

Значения $M(f), N(f),\left|S_{210 С}(N(f))\right|$ для $N\left(f_{\text {в }}\right)=\mathbf{2}$ дБ

\begin{tabular}{|c|c|c|c|c|}
\hline$\Theta_{21 \mathrm{OC}}^{\circ}$ & $M_{2 \text { дБ }}$ & $N_{2 \text { дБ }} \mid$ & $S_{21 \text { ОС }}\left(N_{2 \text { дБ }}(f)\right) \mid$ & $\left|S_{21 \text { ОС }}\left(N_{2 \text { дБ }}(f)\right)\right|$, дБ \\
\hline 180 & 0 & 1 & $\mathbf{2 , 0 0}$ & 6,00 \\
\hline 135 & 0,15 & 1,01 & $\mathbf{2 , 0 2}$ & 6,12 \\
\hline 90 & 0,31 & 1,04 & $\mathbf{2 , 0 9}$ & 6,41 \\
\hline 45 & 0,46 & 1,10 & $\mathbf{2 , 2 0}$ & 6,86 \\
\hline 0 & 0,61 & 1,17 & $\mathbf{2 , 3 5}$ & 7,41 \\
\hline $\mathbf{4 5}$ & $\mathbf{0 , 7 6}$ & $\mathbf{1 , 2 6}$ & $\mathbf{2 , 5 2}$ & $\mathbf{8 , 0 0}$ \\
\hline-90 & 0,92 & 1,36 & $\mathbf{2 , 7 2}$ & 8,07 \\
\hline
\end{tabular}

Та бли ца 3

Значения $M(f), N(f),\left|S_{210 C}(N(f))\right|$ для $N\left(f_{\text {в }}\right)=4$ дБ

\begin{tabular}{|c|c|c|c|c|}
\hline$\Theta_{21 \mathrm{OC}}^{\circ}$ & $M_{4 \text { дБ }}$ & $N_{4 \text { дБ }}$ & $S_{21 \text { ОС }}\left(N_{4 д Б}(f)\right) \mid$ & $\left|S_{21 \text { ОС }}\left(N_{4 \text { дБ }}(f)\right)\right|$, дБ \\
\hline 180 & 0 & 1 & $\mathbf{1 , 5 9}$ & 4,00 \\
\hline 135 & 0,25 & 1,03 & $\mathbf{1 , 6 3}$ & 4,26 \\
\hline 90 & 0,49 & 1,11 & $\mathbf{1 , 7 7}$ & 4,97 \\
\hline 45 & 0,74 & 1,24 & $\mathbf{1 , 9 8}$ & 5,94 \\
\hline 0 & 0,98 & 1,40 & $\mathbf{2 , 2 4}$ & 7,01 \\
\hline $\mathbf{- 4 5}$ & $\mathbf{1 , 2 3}$ & $\mathbf{1 , 5 9}$ & $\mathbf{2 , 5 4}$ & $\mathbf{8 , 0 0}$ \\
\hline-90 & 1,48 & 1,78 & $\mathbf{2 , 8 6}$ & 9,12 \\
\hline
\end{tabular}

Т а бли ц а 4

\begin{tabular}{|c|c|c|c|c|}
\hline$\dot{\circ}_{21 O C}$ & $M_{6 \text { дБ }}$ & $N_{6 \text { дБ }}$ & ||$S_{21 \text { оС }}\left(N_{6 \text { дБ }}(f)\right) \mid$ & $\left|S_{21 \text { ос }}\left(N_{6 д Б}(f)\right)\right|$, дБ \\
\hline 180 & 0 & 1 & 1,26 & 2,00 \\
\hline 135 & 0,34 & 1,06 & 1,33 & 2,5 \\
\hline 90 & 0,69 & 1,22 & 1,53 & 3,72 \\
\hline 45 & 1,04 & 1,44 & 1,82 & 5,20 \\
\hline 0 & 1,38 & 1,70 & 2,16 & 6,68 \\
\hline-45 & 1,73 & 2,00 & 2,53 & 8,00 \\
\hline-90 & 2,07 & 2,30 & 2,91 & 9,30 \\
\hline
\end{tabular}
Значения $M(f), N(f),\left|S_{210 C}(N(f))\right|$ для $N\left(f_{\text {в }}\right)=6$ дБ
На рис. 2 приведены рассчитанные по формуле (2) зависимости коэффициентов отражения $S_{11 \mathrm{Aч} \text { дБ }}$ а на рис. 3 - рассчитанные по формулам (3), (4) зависимости коэффициентов передачи $S_{21 \text { Ач дБ }}$ и номинального коэффициента передачи по мощности

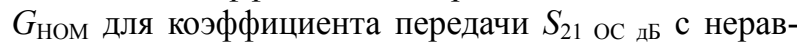
номерности АЧХ 2, 4 и 6 дБ. Зависимости рассчитаны для коэффициента передачи $S_{210 \mathrm{C}}\left(f_{\mathrm{BM}}\right)=8$ дБ и фазы $\Theta_{210 C}=-45^{\circ}$ на верхней частоте для $R C-$ сопротивления параллельной ОС, проводимость которого задается в следующем виде:

$$
Y_{\mathrm{OC} R C}=Y_{\mathrm{OC}}\left[1+j\left(1-\Theta_{21 \mathrm{OC}} / 180^{\circ}\right)\right],
$$

где $Y_{\mathrm{OC}}=1 /\left(1+\left|S_{210 с 0}\right|\right)$.

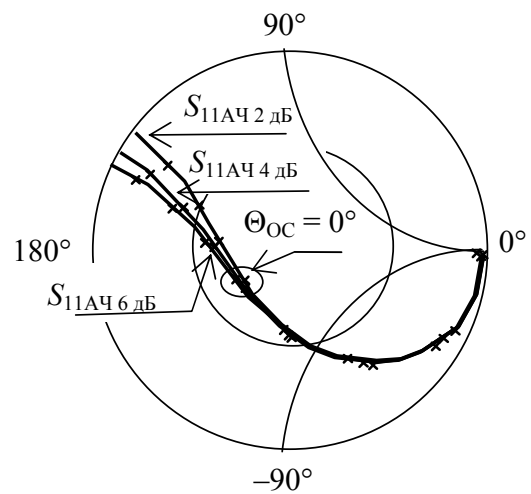

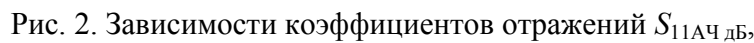
активного четырехполюсника для корректирующего широкополосного усилителя с ОС

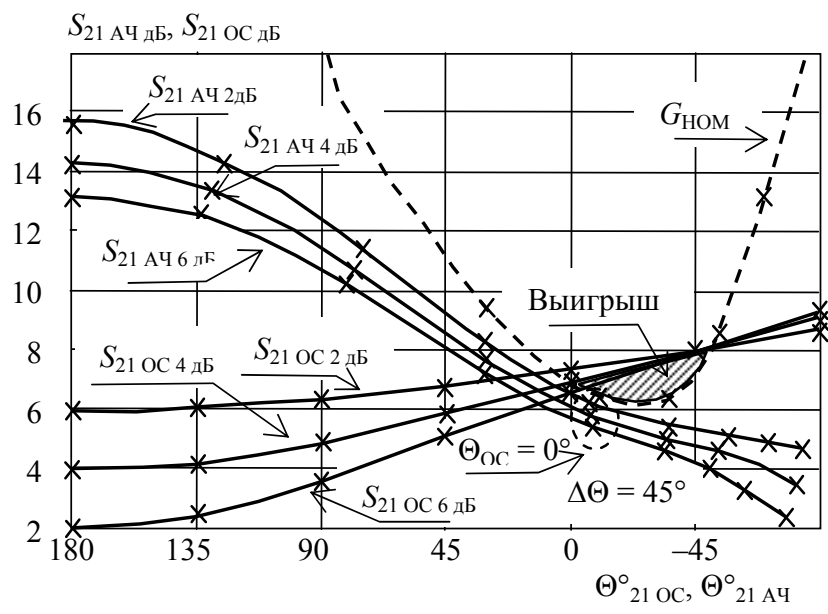

Рис. 3. Зависимости $S_{21 \mathrm{Aч} \mathrm{дь}}$ и $G_{\text {Ном }}$ активного четырех-

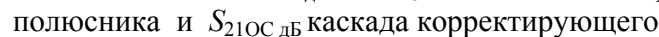
широкополосного усилителя с ОС

Заштрихованная область на рис. 3 показывает выигрыш на верхней частоте коэффициента переда-

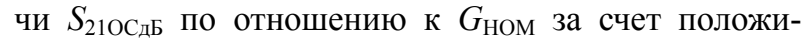
тельной ОС.

\section{Компьютерное моделирование}

Компьютерное моделирование корректирующего широкополосного усилителя с параллельной ОС проведем на основе параметров рассеяния кристалла транзистора КТ3115. Параметры рассеяния кристалла транзистора КТ3115 рассчитаны по эквивалент- 
ной схеме, приведенной на рис. 4, опубликованной в работе [12].

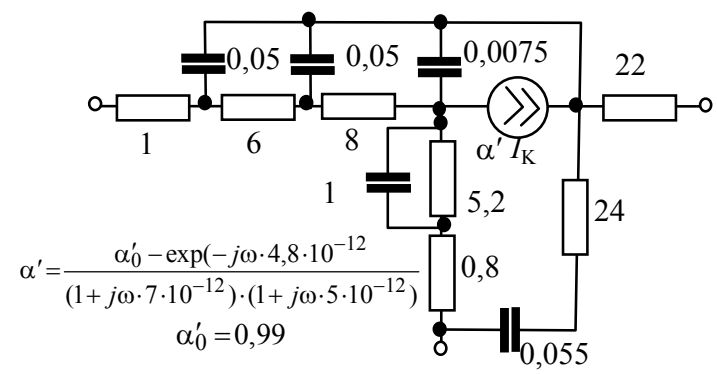

Рис. 4. Эквивалентная схема кристалла транзистора KT3115

На рис. 5 приведены рассчитанные в программе MathCad на основе метода узловых потенциалов зависимости параметров рассеяния кристалла тран-

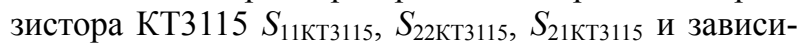

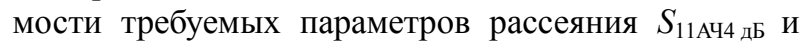
$S_{21 \text { Ач4 дБ }}$ активного четырехполюсника.
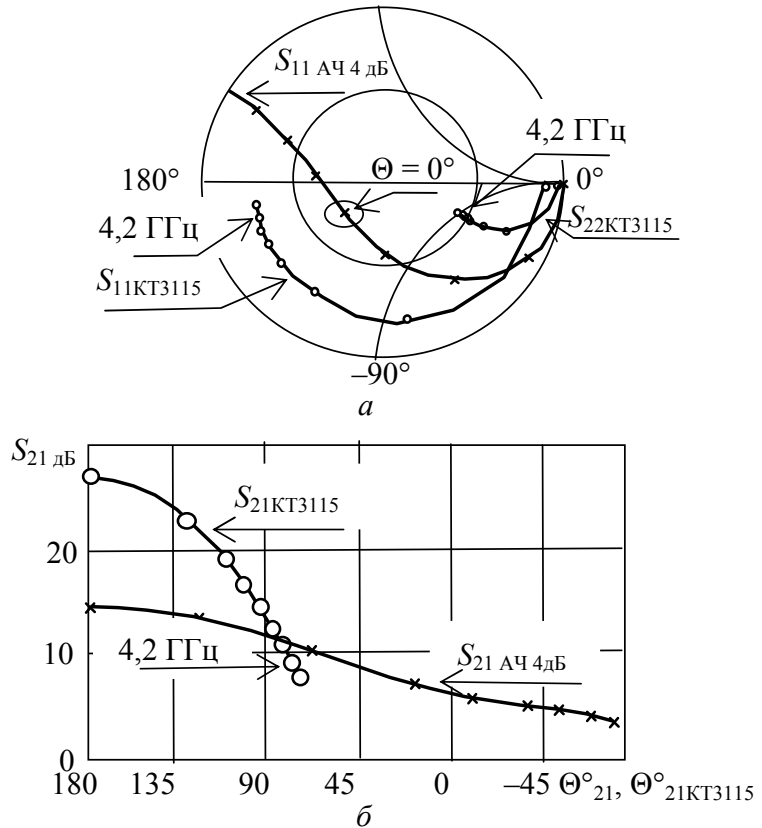

Рис. 5. Зависимости коэффициентов отражения транзистора и требуемого коэффициента отражения $(a)$, коэффициентов передачи транзистора и требуемого коэффициента передачи активного четырехполюсника (б)

Из рис. 5, $а$ видно, что зависимости коэффициентов отражения по входу и выходу транзистора

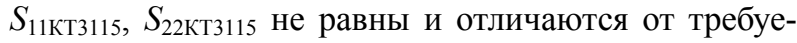
мой зависимости коэффициента отражения активно-

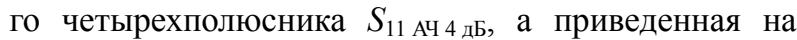
рис. 5, б зависимость коэффициента передачи тран-

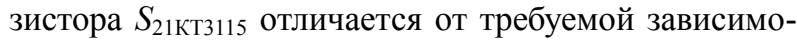
сти коэффициента передачи активного четырехполюсника $S_{21}$ дч 4 дБ по модулю на начальной частоте и по фазе на верхней частоте.

На рис. 6 приведена структурная схема каскада корректирующего широкополосного усилителя с
$\mathrm{OC}$, приближающая параметры рассеяния активного элемента (транзистора) к параметрам рассеяния активного четырехполюсника.

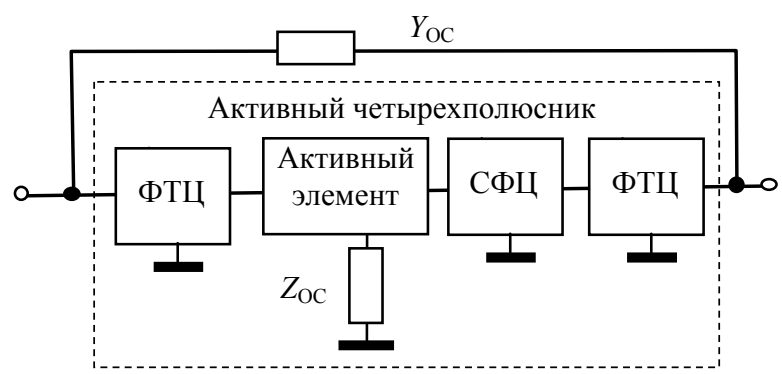

Рис. 6. Структурная схема каскада корректирующего широкополосного усилителя с ОС

Включение двухполюсника последовательной ОС обеспечивает начальный коэффициент передачи активного элемента, СФЦ обеспечивает равенство коэффициентов отражения (2), ФТЦ - значение модуля и фазы коэффициента передачи (3) на верхней частоте.

Для расчета каскада корректирующего широкополосного усилителя с двухполюсником параллельной и последовательной ОС (см. рис. 6) необходимо найти $S$-параметры активного элемента с двухполюсником последовательной ОС, $S$-параметры СФЦ и $S$-параметры ФТЦ.

\section{$S$-параметры активного элемента} с двухполюсником последовательной ОС

В матричном виде алгоритм определения матрицы рассеяния активного элемента с двухполюсником последовательной ОС запишется [13]

$$
\mathbf{S}_{\mathbf{Z}}=-2\left[2\left[-\mathbf{S}_{\text {АЭ}}+\mathbf{E}\right]^{-1}+\mathbf{Z}_{\text {ДОС }}\right]^{-1}+\mathbf{E}
$$

где

$$
\mathbf{S}_{\mathrm{AЭ}}=\left[\begin{array}{ll}
S_{11} & S_{12} \\
S_{21} & S_{22}
\end{array}\right], \mathbf{z}_{\text {ДОС }}=\left[\begin{array}{ll}
Z_{\mathrm{OC}} & Z_{\mathrm{OC}} \\
Z_{\mathrm{OC}} & Z_{\mathrm{OC}}
\end{array}\right], \mathbf{E}=\left[\begin{array}{ll}
1 & 0 \\
0 & 1
\end{array}\right]-
$$

матрицы параметров рассеяния активного элемента; сопротивлений двухполюсника последовательной ОС и единичная матрица.

Тогда матрицу рассеяния активного элемента с двухполюсником последовательной ОС получим в следующем виде:

$$
\begin{gathered}
\mathbf{S}_{\mathbf{Z}}=\left[\begin{array}{ll}
S_{11 Z} & S_{12 Z} \\
S_{21 Z} & S_{22 Z}
\end{array}\right]= \\
=\left[\begin{array}{cc}
\frac{2 S_{11}-Z_{\mathrm{OC}}\left(\Delta S+S_{12}+S_{21}-1\right)}{2-Z_{\mathrm{OC}}(\Sigma S-2)} & \frac{2 S_{12}+Z_{\mathrm{OC}}\left(\Delta S-S_{11}-S_{22}+1\right)}{2-Z_{\mathrm{OC}}(\Sigma S-2)} \\
\frac{2 S_{21}+Z_{\mathrm{OC}}\left(\Delta S-S_{11}-S_{22}+1\right)}{2-\left(Z_{\mathrm{OC}} \Sigma S-2\right)} & \frac{2 S_{22}-Z_{\mathrm{OC}}\left(\Delta S+S_{12}+S_{21}-1\right)}{2-Z_{\mathrm{OC}}(\Sigma S-2)}
\end{array}\right],
\end{gathered}
$$

где $\quad \Delta S=S_{11} S_{22}-S_{12} S_{21}, \quad \Sigma S=S_{11}+S_{12}+S_{21}+S_{22}$, $S_{11 \mathrm{Z}}, S_{12 \mathrm{Z}}, S_{21 \mathrm{Z}}, S_{22 \mathrm{Z}}-S$-параметры активного элемента с двухполюсником последовательной ОС.

Из выражения (6) запишем значение начального коэффициента передачи активного элемента 


$$
S_{21 Z 0}=\frac{2 S_{21}+R_{\mathrm{OC}}\left(\Delta S-S_{11}-S_{22}+1\right)}{2-\left(R_{\mathrm{OC}} \Sigma S-2\right)} .
$$

Для обеспечения требуемого начального коэффициента передачи активного элемента из выражения (7) найдем выражение для сопротивления последовательной ОС

$$
R_{\mathrm{OC}}=\frac{2\left(S_{21 Z 0}-S_{21}\right)}{S_{21 Z 0}(\Sigma S-2)+\Delta S-S_{11}-S_{22}+1} .
$$

Для приближения коэффициента отражения по входу последовательно с сопротивлением ОС вводится индуктивность, которая увеличивает активную составляющую входного сопротивления транзистора [4].

\section{$S$-параметры СФЦ}

Матрица $S$-параметров СФЦ $\mathbf{S}_{\text {СФц }}$ на основе элементов матрицы $s_{11}, s_{12}, s_{21}, s_{22}$ и фазы коэффициента передачи $\Theta_{12}$ СФЦ запишется [14]

$$
\begin{gathered}
\mathbf{S}_{\mathrm{C} \Phi Ц}=\left[\begin{array}{cc}
S_{11 \mathrm{C} \Phi Ц} & S_{12 \mathrm{C} \Phi Ц} \\
S_{21 \mathrm{C} \Phi Ц} & S_{22 \mathrm{C} \Phi Ц}
\end{array}\right]= \\
=\left[\begin{array}{c:c}
s_{11} & \sqrt{1-\left|s_{11}\right|^{2}} \exp \left(i \Theta_{12}\right) \\
\hdashline \sqrt{1-\left|s_{11}\right|^{2} \exp \left(i \Theta_{12}\right)} & -s_{11} \exp \left(i \Theta_{12}\right)
\end{array}\right] .
\end{gathered}
$$

Для последовательного соединения активного элемента с сопротивлением последовательной ОС и СФЦ матрица $S$-параметров запишется [13]

$$
\begin{aligned}
& \mathbf{S}_{\mathbf{Z C} \Phi Ц}=\left[\begin{array}{l:l}
S_{111 Z C \Phi \amalg} & S_{12 Z C \Phi \amalg} \\
S_{21 Z C \Phi Ц} & S_{22 Z C \Phi Ц}
\end{array}\right]=
\end{aligned}
$$

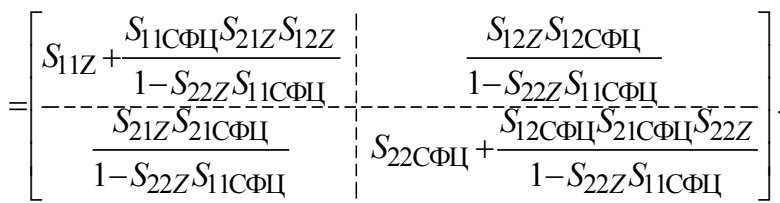

С учетом выражения (9), приравняв коэффициенты отражения СФЦ по входу и выходу в выражении (10), найдем коэффициент отражения по входу СФЦ [15]

$$
s_{11}=\frac{\operatorname{Re}\left\{\left[S_{\mathrm{A}}-\Delta_{S}\right] S_{\mathrm{B}}^{*}\right\}+i \cdot \operatorname{Im}\left\{\left[S_{A}+\Delta_{S}\right] S_{\mathrm{B}}^{*}\right\}}{\left|\Delta_{S}\right|^{2}-1},
$$

где $S_{A}=-\exp \left(i 2 \Theta_{12}\right), S_{\mathrm{B}}=\left[\exp \left(i 2 \Theta_{12}\right) S_{22 Z}-S_{11 Z}\right]$, $\Delta_{S}=S_{11 Z} S_{22 Z}-S_{12 Z} S_{21 Z}, S_{\mathrm{B}}^{*}$ - комплексно-сопряженное значение.

Используя формулы перехода от S-параметров к

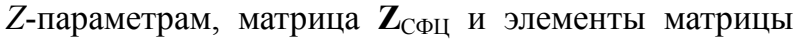
$Z_{11 \text { СФц, }} Z_{12 \text { СФц, }} Z_{21 \text { СФц, }} Z_{22 \text { СФц }} Z$-параметров Т-образной СФЦ (рис. 7) запишутся

$$
\begin{gathered}
\mathbf{Z}_{\mathrm{C \Phi Ц}}=\left[\begin{array}{c:c}
Z_{11 C \Phi \amalg} & Z_{12 \mathrm{C} \Phi \amalg} \\
\hdashline Z_{21 C \Phi Ц} & Z_{22 \mathrm{C} \Phi Ц}
\end{array}\right]= \\
=\left[\begin{array}{c:c}
j \omega L_{1}+1 / j \omega C & 1 / j \omega C \\
\hdashline 1 / j \omega \bar{C} & j \omega L_{2}+1 / j \omega \bar{C}
\end{array}\right],
\end{gathered}
$$

где $L_{1}, C, L_{2}$ - элементы Т-образной СФЦ, $\omega=2 \pi f$.

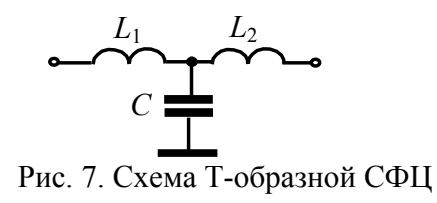

Аналитическое выражение для определения частотно-зависимых $S$-параметров СФЦ в матричном виде запишется [13]

$$
\mathbf{S}_{\mathbf{C \Phi Ц}}=-2\left(\mathbf{Z}_{\mathbf{C \Phi Ц}}+\mathbf{E}\right)^{-1}+\mathbf{E} .
$$

\section{$S$-параметры ФТЦ}

Матрица $S$-параметров ФТЦ запишется [14]

$$
\mathbf{S}_{\text {ФTЦ }}=\left[\begin{array}{c:c}
0 & \exp (-i \Theta) \\
\hdashline \exp (-i \Theta) & 0
\end{array}\right],
$$

где $\Theta$ - фаза ФТЦ на заданной частоте.

Матрица $S$-параметров активного четырехполюсника, приведенного на рис. 6 , запишется

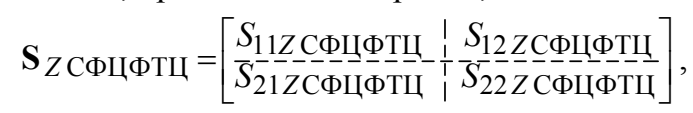

где $\mathbf{S}_{Z \text { СФц Фтц, }} S_{11}$ Z СФц ФтЦ, $S_{21}$ Z СФц ФтЦ, $S_{12}$ Z СФц ФТЦ, $S_{22}$ Z сфц фтц - матрица и элементы матрицы $S$-параметров, полученные для последовательного соединения активного элемента с двухполюсником последовательной ОС (6), СФЦ на выходе (9) и ФТЦ на входе и выходе (14).

В матричном виде матрица рассеяния активного четырехполюсника с двухполюсником параллельной OC (см. рис. 6) запишется [13]

$$
\mathbf{S}_{\mathrm{OC}}=2\left[2\left(\mathbf{S}_{Z \text { СФЦТФЦ }}+\mathbf{E}\right)^{-1}+\mathbf{Y}_{\text {ДОС }}\right]^{-1}-\mathbf{E},
$$

где $\mathbf{Y}_{\text {дОС }}=\left[\begin{array}{cc}Y_{\mathrm{OC}} & -Y_{\mathrm{OC}} \\ -Y_{\mathrm{OC}} & Y_{\mathrm{OC}}\end{array}\right]-$ матрица проводимости двухполюсника параллельной ОС.

Из выражения (16) найдем коэффициент отражения по входу в следующем виде

$$
\begin{aligned}
& S_{11 \mathrm{OC}}=
\end{aligned}
$$

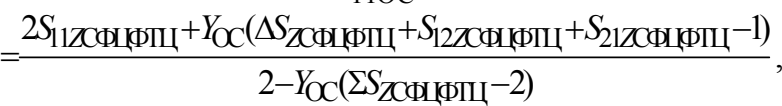

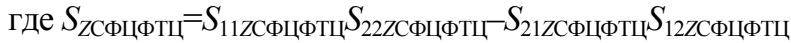

$$
\begin{gathered}
\Sigma S_{Z \text { СФЦТФЦ }}=S_{12 Z \text { СФЦ ТФЦ }}+S_{21 Z \text { СФЦТФЦ }}- \\
-S_{11 Z \text { СФЦТФЦ }}-S_{22 Z \text { СФЦТФЦ. }}
\end{gathered}
$$

Приравняв коэффициент отражения $S_{110 с}$ нулю, найдем выражение для проводимости параллельной OC на верхней частоте [11]

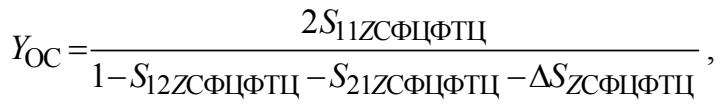

На основе алгоритма математического моделирования корректирующего широкополосного усилителя с двухполюсником параллельной ОС, приведенного автором в работе [11], рассчитаны элементы принципиальной схемы (рис. 8).

В табл. 5 приведены рассчитанные номиналы элементов ФТЦ, СФЦ, двухполюсников параллель- 
ной и последовательной ОС принципиальной схемы каскада корректирующего широкополосного усилителя с ОС, приведенной на рис. 8, для коэффициента передачи 8 дБ на верхней частоте 4,2 ГГц, неравномерности АЧХ 4 и 6 дБ.

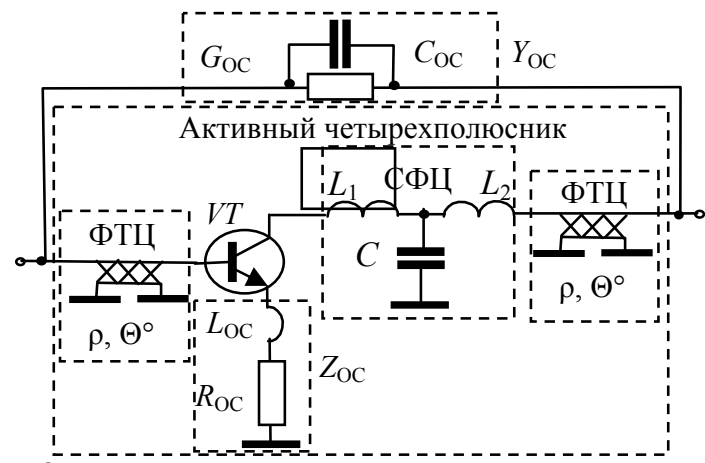

Рис. 8. Принципиальная схема каскада корректирующего широкополосного усилителя с двухполюсником параллельной и последовательной ОС

Т а бли ц а 5

Номиналы рассчитанных элементов ФТЦ, СФЦ, $Y_{O C}, Z_{O C}$

\begin{tabular}{|c|c|c|c|c|c|c|c|c|}
\hline $\begin{array}{c}S_{21 \mathrm{OC}}, \\
\text { дБ }\end{array}$ & $\begin{array}{c}\rho, \mathrm{OM}^{\circ} \\
\Theta^{\circ}\end{array}$ & $\begin{array}{c}L_{1}, \\
\mathrm{H} \Gamma\end{array}$ & $\begin{array}{c}C, \\
\Pi \Phi\end{array}$ & $\begin{array}{c}L_{2}, \\
\mathrm{H} \Gamma\end{array}$ & $\begin{array}{c}1 / G_{\mathrm{OC}}, \\
\text { Ом }\end{array}$ & $\begin{array}{c}C_{\mathrm{OC}}, \\
\Pi \Phi\end{array}$ & $\begin{array}{c}R_{\mathrm{OC}}, \\
\mathrm{OM}\end{array}$ & $\begin{array}{c}L_{\mathrm{OC}}, \\
\mathrm{H} \Gamma\end{array}$ \\
\hline $2-8$ & 50 & 2,6 & 0,42 & 1,6 & 107 & 0,37 & 15 & 1,0 \\
& 14 & & & & & & & \\
\hline $4-8$ & $\begin{array}{c}50 \\
14\end{array}$ & 2,5 & 0,41 & 1,8 & 118 & 0,30 & 12,5 & 1,0 \\
& & & & & & & \\
\hline
\end{tabular}

Анализ номиналов рассчитанных элементов (табл. 5) показывает, что ФТЦ и СФЦ слабо влияют на неравномерность АЧХ в отличие от ФТЦ, СФЦ и $Z_{\mathrm{K}}$ работы [11], где используется корректирующий двухполюсник на входе активного элемента. В данной работе эффективность коррекции АЧХ определяется изменением номиналов сопротивлений ОС.

Введение двухполюсника последовательной ОС, СФЦ и ФТЦ приблизило $S$-параметры транзистора КТ3115 $S_{11 \text { Ктз115 }}, S_{22 \text { Ктз115 }}, S_{21 \text { Ктз115 к требуемым }}$

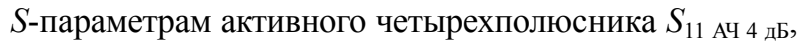

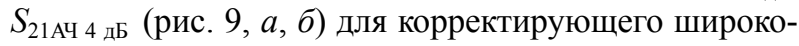
полосного усилителя с нелинейной неравномерностью АЧХ 4 дБ.

Результаты моделирования зависимостей коэффициентов передачи и коэффициентов отражения корректирующих согласованных широкополосных усилителей с двухполюсником параллельной и последовательной ОС с неравномерностью АЧХ в пределах 4-6 дБ для верхних частот 4,2 ГГц приведены на рис. $10, a$ и $б$ соответственно.

\section{Заключение}

Новое аналитическое выражение для коэффициента усиления с учетом нелинейной частотной зависимости АЧХ позволило уточнить математическую модель активного четырехполюсника корректирующего широкополосного усилителя с ОС.

Последовательное сопротивление ОС вместо корректирующего двухполюсника на входе активного элемента с сопротивлением параллельной ОС позволило эффективнее корректировать неравномерность АЧХ широкополосного усилителя с ОС.
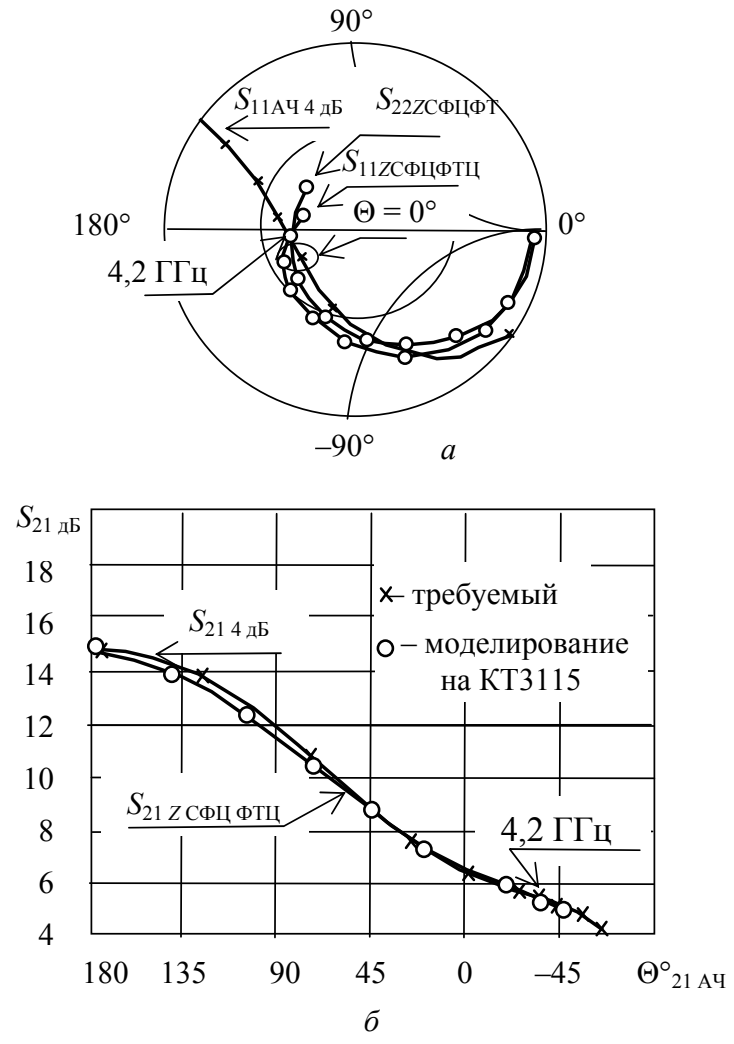

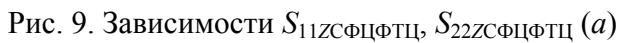

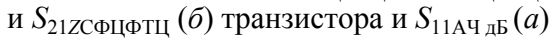

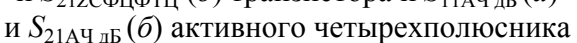

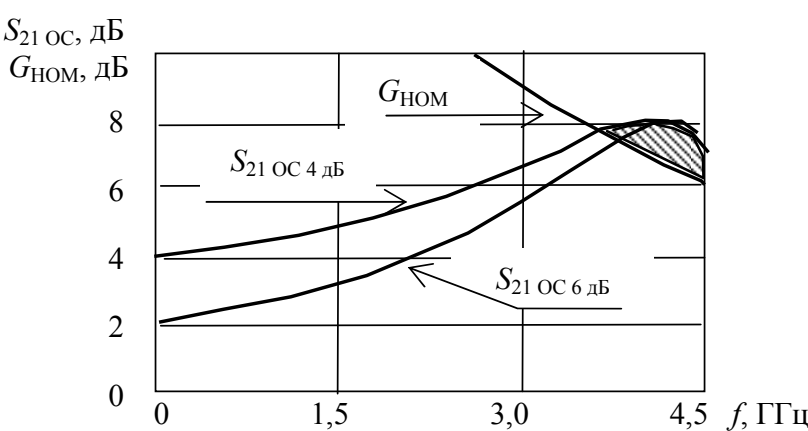

$a$

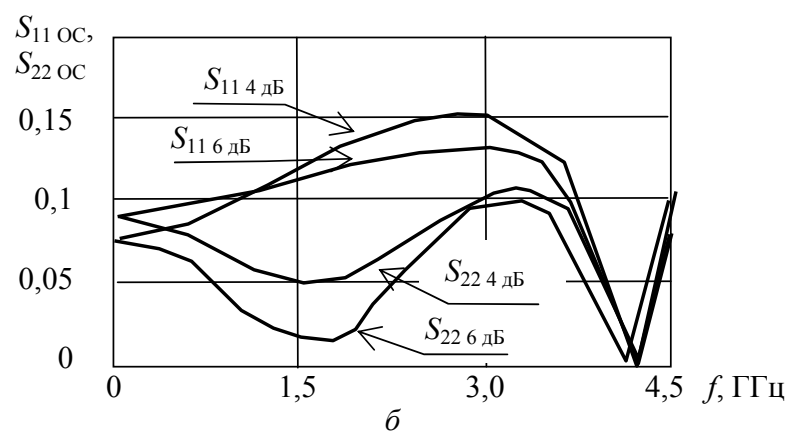

Рис. 10. Зависимости коэффициентов передачи $S_{210 \mathrm{OC} \text { дБ }}, S_{21 \text { ОС д дБ }}(a)$

и коэффициентов отражения $S_{110 с 4 \text { дБ, }} S_{110 с 6 \text { дБ }}($ б) корректирующих широкополосных усилителей с параллельной ОС 
Определен физический смысл (назначение) введенных в структурную схему элементов СФЦ и ФТЦ. СФЦ обеспечивает равенство коэффициентов отражения по входу и выходу транзистора, ФТЦ значение модуля и фазы коэффициента передачи транзистора на верхней частоте.

Приведены результаты расчета элементов принципиальной схемы и результаты компьютерного моделирования коэффициентов передачи и коэффициентов отражения корректирующих широкополосных усилителей с параллельной ОС на кристалле транзистора КТ3115.

\section{Литература}

1. CHA4220-98F A new broadband distributed driver amplifier [Электронный pecypc].- Режим доступа: http: //www.ums-gaas.com/cha4220-98f-a-new-broadbanddist. php (дата обращения: 10.06.2018).

2. 2-22 GHz GaN Low Noise Amplifier [Электронный pecypc]. - Режим доступа: http://www.triquint.com/products/ p/TGA2227 (дата обращения: 22.06.2018).

3. Дроботун Н.Б. Модуль сверхширокополосного усилителя диапазона 10 МГц - 20 ГГц с диссипативной коррекцией АЧХ // Доклады ТУСУР. - 2016. - № 4(19). C. $74-77$.

4. Шварц Н.3. Линейные транзисторные усилители СВЧ. - М.: Сов. радио, $1980 .-398$ с.

5. A new genetic-algorithm-based technique for low noise amplifier synthesis / L.I. Babak, A.A. Kokolov, A.A. Kalentyev, D.V. Garays // 7-th European Microwave Integrated Circuits Conference (EuMIC). - 2012. - P. 381-384.

6. Калентьев А.А. Структурно-параметрический синтез СВЧ-транзисторных усилителей на основе генетического алгоритма с использованием моделей монолитных элементов / А.А. Калентьев, Д.В. Гарайс, И.М. Добуш, Л.И. Бабак // Доклады ТУСУР. - 2012. - № 2(26). - С. 104-112.

7. Бабак Л.И. Автоматизированный синтез СВЧ-транзисторных усилителей на основе генетического алгоритма и использования идеальных трансформаторов импеданса // Доклады ТУСУР. - 2015. - № 3(37). - С. 67-84.

8. An efficient high-frequency linear RF amplifier synthesis method based on evolutionary computation and machine learning techniques / B. Liu, N. Deferm, D. Zhao, P. Reynaert, G. Gielen // Computer-Aided Design of Integrated Circuits and Systems, IEEE Transactions. - 2012. - Vol. 31, No. 7. P. 981-993.

9. Якушевич Г.Н. Математическая модель активного четырехполюсника для широкополосного СВЧ-усилителя с двухполюсником параллельной обратной связи // Доклады ТУСУР. - 2009. - № 2(20). - С. 32-37.

10. Якушевич Г.Н. Алгоритм расчета транзисторного широкополосного усилителя с двухполюсником параллельной обратной связи // Доклады ТУСУР. - 2010.№ 1(21), ч. 2. - С. 63-68.

11. Якушевич Г.Н. Проектирование корректирующего широкополосного усилителя с двухполюсником параллельной обратной связи // Доклады ТУСУР. - 2017. № 3 (20). - С. 144-147.

12. Андрюхов И.П. Кремневый малошумящий биполярный транзистор КТ3115 / И.П Андрюхов, Ю.П. Докучаев, Г.Э. Корнильев и др. // Микроэлектроника и полупроводниковые приборы / под ред. А.А. Васенкова, Я.А. Федотова. - М.: Сов. радио, 1980. - Вып. 5. - С. 43-53.

13. Силаев М.А. Приложение матриц и графов к анализу СВЧ-устройств / М.А. Силаев, С.Ф. Брянцев. - М.: Сов. радио, 1979. -247 с.
14. Фельдштейн А.Л. Синтез четырехполюсников и восьмиполюсников на СВЧ / А.Л. Фельдштейн, Л.Р. Явич. 2-е изд., перераб. и доп. - М.: Связь, 1971. - 388 с.

15. Якушевич Г.Н. Проектирование транзисторных широкополосных усилителей с двухполюсниками параллельной и последовательной обратной связи // Доклады ТУСУР. - 2015. - № 3 (37). - С. 62-66.

\section{Якушевич Геннадий Николаевич}

Канд. техн. наук, с.н.с., доцент каф. радиотехнических систем (РТC) Томского государственного университета систем управления и радиоэлектроники (ТУСУР) Ленина пр-т, д. 40, г. Томск, Россия, 634050

Тел.: 41-37-09

Эл. почта: mrc@main.tusur.ru

Jakushevitch G.N.

Mathematical model of active quadrupole of corrective broadband amplifier with feedback

A mathematical model of an active quadrupole corrective broadband amplifier with non-uniform frequency response (with frequency response increasing with increasing frequency) is obtained, based on a new analytical expression for the gain factor taking into account the nonlinear frequency dependence of the frequency response. Raising the frequency response occurs smoothly with increasing frequency and tends to a linear relationship at high frequencies. For the implementation of non-uniform frequency response is used serial and parallel feedback (OS). The results of computer simulation of the frequency characteristics of a corrective wideband amplifier with non-dimensional frequency response are given. A corrective wideband amplifier can be used to compensate for the unevenness of the frequency response (decrease in frequency response with increasing frequency) of the radio receiving and radio transmitting paths of radio engineering systems.

Keywords: mathematical model, active four-port, correcting, broadband amplifier, OS.

doi: 10.21293/1818-0442-2018-21-4-1-26-32

\section{References}

1. CHA4220-98F A new broadband distributed driver amplifier [Electronic resource]. Access mode: http: www.umsgaas.com cha4220-98f-a-new-broadbanddist. php (accessed: June 10, 2018).

2. 2-22 GHz GaN Low Noise Amplifier [Electronic Resource]. Access mode: http: www.triquint.com products, p TGA2227 (accessed: June 22, 2018).

3. Drobotun N.B. Module ultra-wideband amplifier range $10 \mathrm{MHz}-20 \mathrm{GHz}$ with dissipative correction AFC Proceedings of TUSUR University. 2016, no. 4 (19), pp. 74-77.

4. Shvarts N.Z. Linear transistor amplifiers SHF. [Linear transistor amplifiers SHF], M.: Sov. radio, 1980, 398 p.

5. Babak L.I., Kokolov A.A., Kalentyev A.A., Garays D.V. A new genetic-algorithm-based technique for low-noise amplifier synthesis 7-th European Microwave Integrated Circuits Conference (EuMIC). 2012, pp. 381-384.

6. Kalentiev A.A., Garays D.V., Dobush I.M., Babak L.I. Structural-parametric synthesis of microwave transistor amplifiers based on the genetic algorithm using models of monolithic elements Proceedings of TUSUR University. 2012, vol. 26, № 2, pp. 104-112. 
7. Babak L.I. Automated synthesis of microwave transistor amplifiers based on the genetic algorithm and the use of ideal transformers of impedance Proceedings of TUSUR University. 2015, no. 3 (37), pp. 67-84.

8. Liu B., Deferm N., Zhao D., Reynaert P., Gielen G, An efficient high-frequency linear method based on evolutionary computation and machine learning techniques ComputerAided Design of Integrated Circuits and Systems, IEEE Transactions. 2012, vol. 31, no. 7, pp. 981-993.

9. Yakushevich G.N. A mathematical model of an active four-terminal network for a broadband microwave amplifier with a two-terminal network of parallel feedback. Proceedings of TUSUR University. 2009, no. 2 (20), pp. 32-37.

10. Yakushevich G.N. Algorithm for calculating a transistor broadband amplifier with a double-pole of parallel feedback. Proceedings of TUSUR University. 2010, no. 1 (21), h. 2 , pp. 63-68.

11. Yakushevich G.N. Designing a correcting broadband amplifier with a two-pole of parallel feedback. Proceedings of TUSUR University. 2017, no. 3 (20), pp. 144-147.

12. Andryukhov I.P.,. Dokuchaev Yu.P., Korniliev G.E. et al. Silicon low-noise bipolar transistor KT3115. Microelectronics and Semiconductor. Devices Ed. A.A. Vasenkova, Ya.A. Fedotov, M.: Sov. Radio, 1980, no. 5, pp. 43-53.
13. Silaev M.A. Prilozhenie matric $i$ grafov $k$ analizy CVCH yctroictv [Application of matrices and graphs to the analysis of microwave devices] M.A. Silaev, S.F. Bryantsev. M.: Sov. radio, 1979, 247 p.

14. Feldstein A.L., Javiw L.R.. Cintez chetyrehpolucnikov $i$ vocmipolucnikov na $\mathrm{CVCH}$. [Synthesis of fourterminals and eight-ports on microwave frequencies] 2 nd ed., Revised. and additional. M.: Communications, 1971, 388 p.

15. Yakushevich G.N. Designing of transistor broadband amplifiers with two-ports of parallel and sequential feedback. Proceedings of TUSUR University. 2015, no. 3(37), pp. 62-66.

\section{Gennady N. Jakushevitch}

Candidate of Engineering, Assistant Professor, Department of Radio Engineering Systems, Tomsk State University of Control Systems and Radioelectronics (TUSUR) 40, Lenin pr., Tomsk, Russia, 634050

Phone: 41-37-09

Email:mrc@main.tusur.ru 